\title{
Dental age estimation in children and adolescents
}

\section{Abstract}

Age estimation, using forensic odontology, is a crucial step for biological identification Nowadays, numerous methods are available for estimating age in various stages of life using the dentition. Amongst the many known methods till date Demirjian's method is the most widely used.

\author{
Volume 9 Issue 4 - 2018 \\ Garima Rawat, Kirti Kureel \\ Dharamshila Narayana Cancer Hospital, India
}

Correspondence: Garima Rawat, Dharamshila Narayana Cancer Hospital, New Delhi-I I0002, India,Tel +9|-9873776634,

Email garima3103@gmail.com

Received: May 29, 2018 | Published: July 09, 2018

\section{Introduction}

Forensic Odontology is the branch of dentistry which in the interest of justice deals with the proper handling and examination of dental evidence, and with the proper evaluation and presentation of dental findings. Forensic is derived from the Latin word forum which means "court of law". Odontology refers to the study of teeth. ${ }^{1}$

Age estimation forms an important part of forensic medicine and odontology for identification of deceased victims and also for crimes and accidents. A forensic age estimate of a living person for the purpose of criminal prosecution should consist of: a physical examination that also records anthropometric data, any age-relevant developmental disorders and signs of sexual maturation, an X-ray examination of the left hand, dental examination that records dentition status and evaluates an orthopantomograph. ${ }^{1}$ Age estimation can be done by using teeth, ossification of bones, height and weight data and miscellaneous data.

There are various methods available for estimating age. ${ }^{2}$

According to the state of development of the dentition: ${ }^{3}$

1. Forming dentition

2. Adult fully formed dentition.

According to the technique of investigation: ${ }^{3}$

1. Clinical or visual

2. Radiographic

3. Histological

4. Physical and chemical analysis

Age estimation using dentition can be categorized into three phases: ${ }^{3}$

a. Age estimation in prenatal, neonatal and early postnatal child

b. Age estimation in children and adolescents

c. Age estimation in adults

Odontology might provide solution with the help of specific techniques depending on the fact whether the individual is a child or an adult. ${ }^{2}$

During this phase of age estimation the parameters used are "eruption" \& "calcification". Although these are better analysed in the deciduous dentition as it is more regular and timed (6 m-2.5yr).
On the contrary, in the permanent dentition, there is influence of various factors which play a role like the intraoral environment, arch space, infection and premature tooth loss. Amongst, eruption and calcification, it is better to evaluate and estimate age by "calcification" as the teeth can be followed over a period of years rather than months or days, not altered by local factors and assessment of age is possible even during the period when no teeth erupt. ${ }^{2-4}$

Methods for age estimation in children and adolescents include: ${ }^{3-5}$

1. Schour and Massler's method (1941)

2. Nolla's method (1960)

3. Morrees, Fanning and Hunt method (1963)

4. Demirjian's method (1973)

5. Open apex method (Cameriere method)

6. $3^{\text {rd }}$ molars

\section{Schlour and massler method}

These authors chart/atlas was the $1^{\text {st }}$ attempt at scientific dental age estimation which was based on survey. The chart is based on direct comparisons with radiographs. They gave 20 stages of tooth development from 4 months in utero to 21 years of age. These charts do not have separate surveys for males and females. The charts have been improved by Ubelaker. ${ }^{6}$

\section{Nolla's method}

In this method, the mineralization of permanent dentition was divided in ten stages. The radiograph of patient is matched with a comparative figure and can be used to assess the development of each tooth of the maxillary and mandibular arch. Each tooth is given a reading, totalling is made and the total I compared with the table provided by Nolla. The advantages of this method are that it can be applied to an individual with or without the third molar and that girls and boys are dealt with separately. ${ }^{6}$

\section{Morrees, fanning and hunt method}

They developed 14 stages of mineralization for developing single and multicoated permanent teeth. Published charts based on a radiographic survey that give details of both deciduous and permanent dentition development. Specimen radiographs should be compared against the charts. The earliest age in the survey was 6 months and the data also include the development of the third mandibular molar. Separate charts for each sex. Range between \pm two standard deviations 
represent an age range in which $95 \%$ of the population would be expected to reach the appropriate developmental landmark. ${ }^{6}$

\section{Demirjians' method}

This is the most common method for age estimation and was published in 1973 by Demirjan, Goldstein and Tanner. Originally, Demirjian studied development of seven left mandibular teeth which was divided into 8 stages (A to $\mathrm{H}$ ).

Later on it was modified by other researchers and $3^{\text {rd }}$ molar was included. They proposed this method that makes use of ten developmental stages (numbered 0-9) for the eight left mandibular permanent teeth.

Based on the developmental stage of each tooth, a sex-specific 'maturity score' is assigned to them. The maturity score allocated to the teeth is added and a total maturity score obtained. This total is then substituted in a formula. Keeping in mind the differences in the dental development of females and males, separate maturity scores and formulas were provided for males and females.

To simplify comparison, the developmental stages are defined by certain criteria. Each developmental stage may have one, two or three criteria marked (a), (b) and (c).

1. If only one criterion is given, this must be observed on the radiograph in order to select the stage

2. If two criteria are given, the first one must be observed on the radiograph in order to select the stage

3. If three criteria are given, the first two of them must be observed on the radiograph for the stage to be selected.

In borderline cases, the earlier stage is always considered.

Stage 0

a. Dental calcification has not yet begun.

Stage 1

a. The bone crypt has formed, but no sign of the tooth germ.

Stage 2

a. In both uniradicular and multiradicular teeth, a beginning of calcification is seen at the superior level of the crypt, in the form of inverted cone or cones. There is no fusion of these calcified points.

Stage 3

a. Fusion of the calcified points forms one or several cusps, which unite to give a regularly outlined occlusal outline.

Stage 4

a. Enamel formation is complete at the occlusal surface. Its extension and convergence towards the cervical region is seen.

b. The beginning of dentinal deposit is seen.

c. The outline of the pulp chamber has a curved shape at the occlusal border.

Stage 5 a. The crown formation is completed down to the cemento-enamel junction.

b. The superior border of the pulp chamber in uniradicualr teeth has definite curved form, being concave towards the cervical region. The projection of the pulp horns, if present, gives an outline like an umbrella top. In molars, the pulp chamber has a trapezoidal form.

c. Beginning of root formation is seen in the form of a spicule.

Stage 6

Uniradicular teeth

a. The walls of the pulp chamber now form straight lines, whose continuity is broken by the presence of the pulp horn, which is larger than in the previous stage.

b. The root length reaches at least $1 / 3$ rd of the crown height.

Multiradicular teeth

a. Initial formation of the radicular bifurcation is seen in the form of either a calcified point or semi-lunar shape.

b. The root length reaches at least $1 / 3$ rd of the crown height.

Stage 7

Uniradicular teeth

a. The walls of the pulp chamber now form a more or less isosceles triangle. The apex ends in a funnel shape.

b. The root length is equal to or greater than the crown height.

Multiradicular teeth

a. The calcified region of the bifurcation has developed further down from its semi-lunar stage to give roots a more definite and distinct outline, with funnel shaped endings.

b. The root length is equal to or greater than the crown height.

Stage 8

a. The walls of the root canal are now parallel (distal root in molars).

b. The apical ends of the root canals are still partially open (distal root in molars).

Stage 9

a. The apical end of the root canal is completely closed (distal root in molars).

b. The periodontal membrane has a uniform width around the root and apex.

The advantages of this method were it is simple to use, detailed description provided and there is radiographic illustration of the developmental stages. However, this method causes overestimation of age and the formulas are cannot be used worldwide. Due to this, country specific formulas have been developed by researchers. India-specific regression formulas have been developed which give better age estimates than the original formulas. This suggests that Demirjian's 8-teeth method also needs adaptation prior to use in diverse populations. ${ }^{6,7}$ 


\section{Camiriere method}

Formulated a linear regression formula for assessing the chronological age utilizing the measurements of open apices in teeth. Seven lower teeth (excluding the $3^{\text {rd }}$ molars) from the left side were used in the Italian population. Ratio of the height of the teeth and width of their 'open' apex is measured; this allows compensating for magnification and angulation errors that may have been induced during radiography. ${ }^{8}$

\section{Third molars for age estimation}

Third molars can be useful indicators of age between 16 to 23 years of age. However, due to immense variations in genesis, position, morphology and time of formation the accuracy is questionable. Gunst and his co-workers assessed third molars in Belgian Caucasian individuals and they concluded that if all four third molars are calcified, chances of being 18 year old are $96.3 \%$ in Males and $95.1 \%$ Females. Also, lower $3^{\text {rd }}$ molars are better indicators in case all four third molars haven't erupted. ${ }^{9}$

\section{Conclusion}

Therefore, age estimation plays an important role in forensic odontology. Although, it is a challenging task still sufficient research supports its valuability. The main objective is to obtain the best standardized method for legal, medical age estimation, which is reproductive, simple and reliable, that we can apply in living and dead.

\section{Acknowledgement}

None.

\section{Conflict of interest}

The authors declare that there is no conflict of interest.

\section{References}

1. Divakar KP. Forensic Odontology: The New Dimension in Dental Analysis. Int J Biomed Sci. 2017;13(1):1-5.

2. Duangto P, Janhom A, Prasitwattanaseree S, et al. Age Estimation Methods in Forensic Odontology. Journal of Dentistry Indonesia. 2016;23(3):74-80.

3. Priyadarshini C, Puranik MP, Uma SR. Dental Age Estimation Methods: A Review. Int J Adv Health Sci. 2015;1(12):19-25.

4. Gupta $\mathrm{S}$, Mehendiratta $\mathrm{M}$, Rehani $\mathrm{S}$, et al. Age estimation in Indian children and adolescents in the NCR region of Haryana: A comparative study. J Forensic Dent Sci. 2015;7(3):253-2588.

5. Nayak SD, George R, Shenoy A, et al. Age Estimation in Forensic Dentistry- A Review. International Journal of Scientific Research. 2014;3(4):333-338.

6. Panchbhai AS. Dental radiographic indicators, a key to age estimation. Dentomaxillofac Radiol. 2011;40(4):199-212.

7. Acharya $\mathrm{AB}$. Age estimation in Indians using Demirjian's 8-teeth method. J Forensic Sci. 2011;56(1):124-127.

8. Pratyusha K, Prasad MG, Radhakrishna AN, et al. Applicability of Demirjian's Method and Modified Cameriere's Methods for Dental Age Assessment in Children. Journal of Clinical and Diagnostic Research. 2017;11(2):ZC40-ZC43.

9. Gunst K, Mesotten K, Carbonez A. Third molar root development in relation to chronological age: a large samplesized retrospective study. Forensic Sci Int. 2000;136(1-3):52-57. 